\title{
Gestión de documentos técnicos: una proyección en la Universidad del Zulia
}

\author{
Ana Judith Paredes Chacin \\ Neyda Paredes Chacin \\ Universidad del Zulia - LUZ, República Bolivariana de Venezuela
}

CASE REPORTS

\begin{abstract}
Resumen
Objetivo. Analiza de forma integral y sistemática los principios de organización y procedimiento técnico, que respaldan la gestión de documentos basada en el uso de las tecnologías de información. Método. Se desarrolló un estudio de campo basado en el método descriptivo-documental, en el contexto de la Gerencia de Proyectos de la Dirección de Infraestructura (Dinfra) de la Universidad del Zulia. Resultados. En los resultados obtenidos es evidente la eficiencia de los procesos que respaldan la gestión de documentos técnicos: planos, cálculos métricos y memorias descriptivas generados en la Dinfra. Conclusión. Los fundamentos conceptuales, de organización y técnica archivística, contribuyen con la sistematización, el reguardo y la preservación documental, además de garantizar la recuperación oportuna de la información técnica para la gestión de la Universidad del Zulia.
\end{abstract}

Palabras clave

Gestión de documentos técnicos; Procesos de archivos; Sistemas de información; Fundamentos organizacionales; Universidad del Zulia; República Bolivariana de Venezuela

\section{Management of technical documents: a projection at the University of Zulia}

\begin{abstract}
Objective. This paper analyze comprehensively and systematically the principles of organization and technical procedure, which support document management based on the use of information technologies. Method. We developed a study based on the documentary descriptive method in the context of the Dirección de Infraestructura (Dinfra) from the Universidad del Zulia. Results. We find evidence of efficiency in the processes that support the management of technical documents: Plans, metrics and memories generated by the Dinfra. Conclusion. The conceptual basis of organizational and technical archives contribute to the systematization, shelter save and documental preservation, and ensure the timely retrieval of technical information for the management of the University of Zulia.
\end{abstract}

Keywords

Management technical documents; Arquives processes; Information systems; Organizational concepts; Universidad del Zulia; República Bolivariana de Venezuela

\section{Introducción}

Los procesos inherentes con la gestión de información y el conocimiento se encuentran en constantes avances y desarrollos, sobre todo en el contexto de las instituciones universitarias venezolanas, lo cual queda evidenciado al observar las diversas formas y medios tecnológicos, mediante los cuales se accede a los servicios de información.

El alcance de lo expuesto, se proyecta para soportar las funciones de docencia, investigación y extensión, así como también las que se ejercen en las dependencias administrativas, donde de forma continua buscan adaptarse a las transformaciones necesarias para alcanzar eficientemente sus objetivos, como es el caso de la Dirección de Infraestructura de la Universidad del Zulia (Dinfra-LUZ), considerada como objeto de estudio de la presente investigación. 
Dependencia administrativa adscrita al Vicerrectorado Administrativo de LUZ, la cual en búsqueda del mejoramiento continúo y de la dinamización de su gestión apoya el objetivo de analizar los principios organizacionales y técnicos procedimentales para consolidar la gestión de los documentos técnicos que genera como parte de sus funciones rectoras. Esta iniciativa, se apoya en la necesidad de viabilizar la aprobación de la Unidad de Gestión Documental Técnica de Infraestructura, con la correspondiente consecución de los recursos, para cumplir con la función de interactuar con todos los usuarios reales y potenciales, así como contribuir con el apoyo de la toma de decisiones de profesionales y técnicos que lideran estratégicamente el desarrollo armónico de la infraestructura de la Universidad del Zulia (LUZ).

\section{Avances y desarrollos en gestión técnica documental}

En el ámbito local y global la gestión de documentos, así como la sistematización de la información respaldada por el uso de las tecnologías de información y comunicación (TIC), se conciben como recursos de trascendencia para impulsar el desarrollo funcional de las organizaciones cuyo quehacer se fundamenta en la producción de recursos informativos, los cuales requieren de la aplicación de estándares y técnicas para su efectivo tratamiento en pro de garantizar la accesibilidad de la información y el conocimiento plasmado indistintamente cual sea su formato.

Desde esta perspectiva, se destaca el desarrollo alcanzado en las instituciones universitarias venezolanas, específicamente en la Universidad del Zulia, mediante las iniciativas del Sistema de Archivo e Información (SAILUZ), se han definido lineamientos para procesar y sistematizar la producción documental de índole académico-administrativo que soporta la trayectoria de esta universidad. En el caso de la Dirección de Infraestructura Dinfra, se establece de forma preliminar la efectividad de sistemas y procesos técnicos archivísticos que para la actualidad han garantizado, no sólo el procesamiento y almacenamiento de los documentos administrativos, sino también su preservación, así como su oportuna recuperación, sin embargo la adaptabilidad de tendencias centradas en software y hardware forma parte de las acciones por emprender.

Se prevé, que la definición de acciones para el procesamiento de la colección de documentos técnicos: planos y sus soportes descriptivos, han de contribuir desde una perspectiva técnica-operativa a superar debilidades que impactan en la institución producto del:

- crecimiento acelerado de la producción técnica documental (planos, expedientes técnicos)

- necesidad de estandarizar el uso de sistemas de gestión técnico documental desde una perspectiva integral y soportado mediante el uso de las tecnologías de información, considerando para los efectos la naturaleza y fases de producción,

- débil proyección hacia la sistematización y digitalización de la colección centralizada que datan desde el año 1964 hasta la actualidad.

- manipulación excesiva de documentos originales que impiden promover su preservación.

- inexistencia de un Repositorio de Documentos Técnicos, que permita almacenar de forma sistémica los planos disponibles en formato digital.

- debilidades estructurales para consolidar organizacional y funcionalmente la Unidad de Gestión Técnico Documental de la Dirección de Infraestructura

- $\quad$ necesidad de resguardar y promover el valor informacional y patrimonial para la institución y sectores relacionados, sobre tan importante colección.

En función de los aspectos descritos, se fundamenta la necesidad de afianzar los componentes organizacionales y técnicos procedimentales, los cuales según Zambrano (2005) expone que el logro de avances en lo referente con la gestión de información, permitirá contribuir con la necesidad de instaurar de forma sistemática procesos de gestión efectivamente planificados, organizados y eficientes, así como promover la credibilidad, pertinencia, calidad, seguridad, confiabilidad y estabilidad de los servicios de información. 


\section{Principios para la gestión de documentos}

La consolidación del proceso de transformación organizacional de la Dirección de Infraestructura, fundamenta en la actualidad que su Unidad de Gestión Operativa, conocida como la planoteca, se proyecte mediante la efectiva aplicación de los principios que se describen a continuación, lo cual permitirá afianzar sus funciones principales, como la centralización y procesamiento de documentos técnicos altamente demandados por los usuarios.

\subsection{Principio organizacional}

El crecimiento y consolidación de la Dirección de Infraestructura y por ende la expansión de sus funciones, fundamenta que se constituya la denominada planoteca de la Universidad del Zulia, como la Unidad de Gestión Documental Técnica de Infraestructura. Esta se proyecta como la unidad de información especializada, regida mediante una visión funcional y operativa, cuya interacción con las instancias correspondientes, le permita contribuir de forma directa con el desarrollo y cumplimiento de las competencias centradas en el servicio y sistematización de la documentación técnica generada en la institución.

Para tales efectos, se prevé la interacción funcional con el Sistema de Archivo e Información de la Universidad del Zulia (Sailuz/Cedia: unidad central), así como la relación de dependencia administrativa con la Dinfra y sus Departamentos: Planificación Física, Proyectos, Construcción de Obras, Mantenimiento de Infraestructura y Ambiente, cada una de estas gerencias son generadoras de altos volúmenes de información técnica producto del desempeño de sus actividades, y por ende se requiere del fortalecimiento de la gestión de información en el ámbito universitario. Para los efectos, se visualiza en gráfico 1, la estructura matricial que proyecta la relación funcional entre los programas y procesos de gestión concebidos para el óptimo funcionamiento de la Unidad de Gestión Documental Técnica Informativa de la Dinfra.

\section{Gráfico 1. Estructura organizativa matricial de la Unidad de Gestión Documental Técnica-DINFRA LUZ}

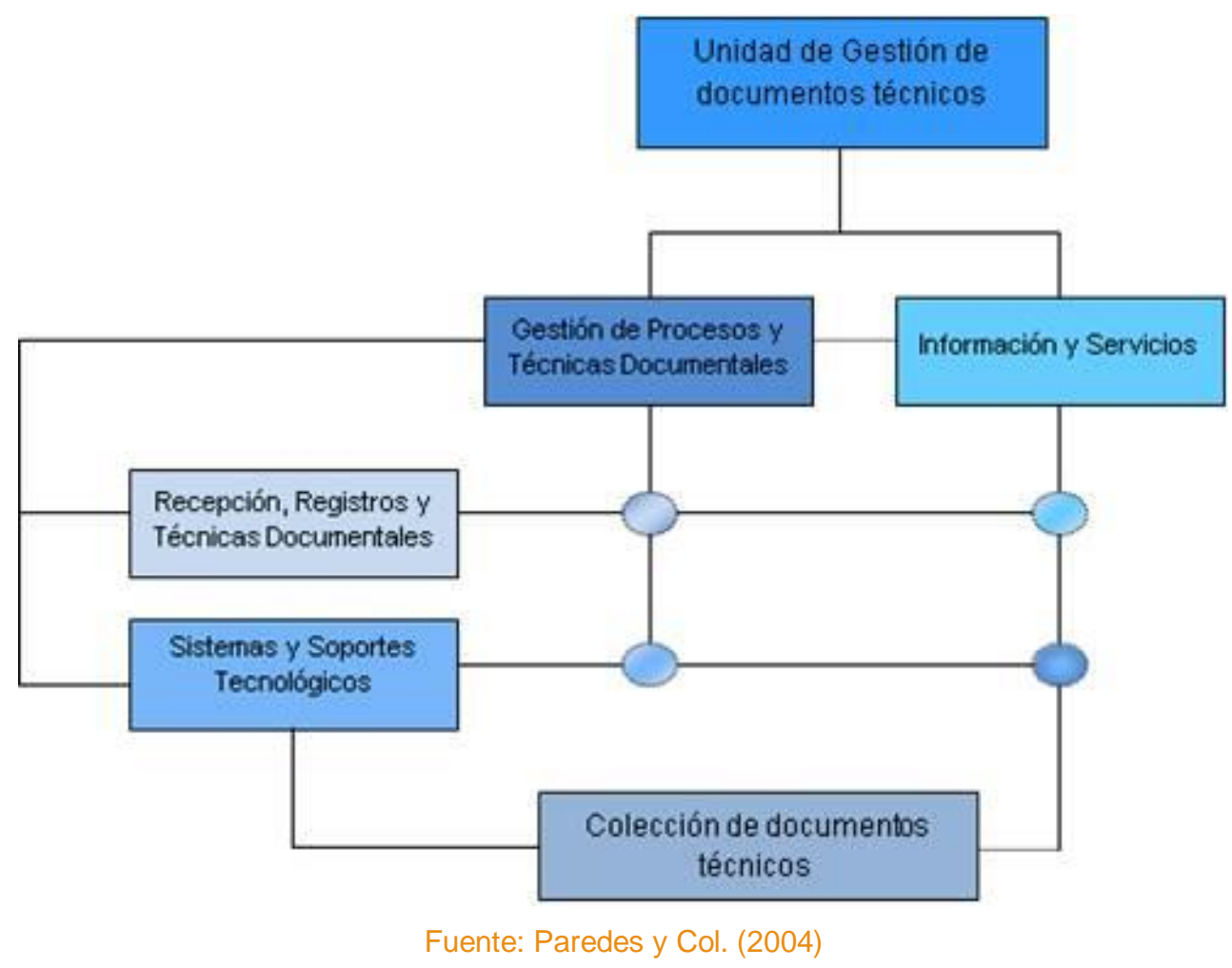

La estructura propuesta, ha de soportar la interacción entre cada uno de los programas y procesos definidos, y se fundamenta en la organización aprobada y concebida en el Proyecto de transformación del Sistema de Archivo e Información de LUZ (2004). En tal sentido, la viabilidad de la estructura presentada, se concibe integralmente para responder y dar fiel cumplimiento de las funciones técnicas archivísticas que se aplican desde el momento de la producción de los documentos técnicos: proyectos, planos, memorias descriptivas, en pro de garantizar la efectiva accesibilidad y recuperación de información para los usuarios de los cuales se 
mencionan: arquitectos, ingenieros, personal técnico y de apoyo, estudiantes, docentes e investigadores, dependencias centrales, facultades, núcleos de LUZ y usuarios de organismos gubernamentales.

En este orden de ideas, se hace referencia desde la perspectiva conceptual basada en los principios de gestión, por los cuales ha de regirse la unidad objeto de estudio que según lo aprobado en el proyecto de modernización organizacional y funcional del Sistema de Archivo e Información de LUZ(2004), que su Misión, ha sido concebida en pro de liderar los procesos de gestión de información, así como la socialización del conocimiento institucional; a través, de la centralización y preservación de la producción documental, la operatividad de sistemas como también el uso de nuevas tecnologías de información y comunicación; para ello, dispone de estructuras dinámicas, flexibles y potencialidades humanas que promueven y apoyan el desarrollo de la universidad y de los sectores socioeconómicos del país.

Con respecto de su Visión, se concibe como la unidad capaz de promover la socialización del conocimiento institucional, a través de la gestión informativa documental soportada por el uso de las Tecnologías de Información y Comunicación (TIC).

\subsection{Principio técnico-procedimental que rigen la gestión de documentos}

En el marco de lograr la consolidación de la gestión técnica documental, así como considerando el principio conceptual definido por la "Guide to the Archival Care of Architectural Records 19 th- 20 th Centuries" (2000), la planoteca se concibe como el lugar donde se guardan los documentos vinculados con proyectos de obras, memorias descriptivas, planos arquitectónicos de iglesias, jardines, edificios y maquetas relacionadas con el tipo de proyecto. Es decir, se define a la colección de planos como la representación gráfica, detallada y exhaustiva de todos los elementos que plantea un proyecto arquitectónico o civil constructiva, en forma que los define completamente en sus tres dimensiones: lineales, superficiales y volumétricas.

Colección que amerita de la aplicación de procesos técnicos y sistemas especializados que garanticen el flujo de información, razón por la cual se analizan conceptualmente los principales fundamentos teóricos, así como su relación con los sistemas de información desarrollados en la institución y aplicados en una primera fase, los cuales operativamente soportan el quehacer y la sistematización de la colección técnica documental, según se especifican a continuación.

\subsubsection{Gestión Técnica Archivística}

Previo a la especificación de los principios que rigen la gestión técnica documental, se analiza uno de los componentes fundamentales que se basa en la necesidad de identificar la tipología de los documentos, es por ello que en el caso objeto de estudio la denominada: Colección de Documentos Técnicos (CDT), ha sido conceptualmente definida como un conjunto artificial de documentos acumulados sobre la base de alguna característica común, sin tener en cuenta su procedencia. En tal sentido, no debe confundirse con el fondo. Según se expone, la procedencia del fondo documental es producto reunido o utilizado en torno a las actividades y funciones de su productor, la colección reúne piezas que comparten características comunes, ya sea una materia, ya sea un tipo documental concreto. (General International Standard Archival Descriptions ISAD (G). 2000, p. 16-17).

De acuerdo con lo descrito, la formación de las colecciones técnicas contenidas en la planoteca, dio su inicio en la antigua Junta de Planificación Física creada a partir de 1975, pero su evolución como dependencia y transformación en 1999, promovió el crecimiento del fondo y por ende generó cambios que se revelan en la conformación del actual fondo documental técnico cuya naturaleza técnica - descriptiva, ha permitido constituir diversas colecciones documentales que soportan los proyectos de infraestructura de la Universidad del Zulia.

Colección integrada por: memorias descriptivas, especificaciones técnicas, cómputos métricos, presupuestos, análisis de costos, cargas térmicas según el tipo de proyecto y los planos respectivos integrados en un solo expediente pero existe además: serie documental gráfica o planos y una serie documental escrita o de memorias descriptivas separadas; colección hemerográfica, referencial, bibliográfica, catálogos, fotografías, así como material cibernético o digital.

Asimismo, existe una serie documental referencial constituida por leyes, normas, reglamentos, entre otros, por lo cual se destaca que operativamente, desde el año 2004, la organización de la documentación técnica, se ha 
ejercido considerando parte de los principios archivísticos: el criterio de origen de procedencia clasificando planos y proyectos según su origen, dependiendo de la facultad o dependencia de donde proviene.

\subsubsection{Clasificación Documental:}

Proceso estratégico el cual ha sido concebido, previa aplicación del Sistema de clasificación documental administrativo SICDA, elaborado desde el Sistema de Archivo e Información de LUZ, regido por el principio de origen y procedencia. De esta forma, se cumple con lo expresado por Mendo (2004) quien considera que al hablar de los principios de la archivología, se amerita considerar la procedencia y orden original; así como, del ciclo vital hace referencia a que éstos establecen y dirigen el tratamiento del documento a lo largo de toda su vida, consolidando las operaciones archivísticas que son propias en cada una de las fases. Asimismo, el desempeño en el procesamiento técnico se lleva a efecto mediante una variante del Sistema de Documentación Técnica (SIDOTEC) propuesto por el SAILUZ y la adaptación de criterios utilizados en la unidad, derivados de la misma tipología documental existente.

\section{Tecnologías y sistemas de información para la gestión de documentos técnicos}

En la actualidad la dinamización de la gestión de las Unidades de Información conocidas estas como: Bibliotecas, Archivos, así como los Centros de Documentación, se rigen en su mayoría por la asertividad de los programas tecnológicos concebidos para llevar a efecto el denominado proceso de sistematización informacional.

En tal sentido, el diseño de sistemas de información (desarrollo de software: sistemas operativos, memoria interna, almacenamiento, calidad de visualización, capacidad de las redes y velocidad), es considerado como una de las fases mediante la cual, se requiere del análisis procedimental, así como se propone la asignación y el soporte de la plataforma (hardware: computadores, impresoras, escáneres o cámaras digitales) que garantiza el registro automatizado desde una perspectiva de gestión administrativa (registro y procesos archivísticos), hasta la fase de digitalización y recuperación de la información plasmada en la colección de los documentos técnicos. Sin dejar de considerar que para la estructura del diseño del sistema de información, se prevé su desarrollo en ambientes de software libre e interoperables, previo análisis de los requerimientos de los usuarios.

Por otra parte, se menciona la necesidad de consolidar el Repositorio de Información Documental Técnica, cuyos datos e imágenes se soportan como documentos en soporte digital, con estándares de calidad que permitan: estandarizar parámetros: definir resolución, números de bits, escala de colores o grises, control de calidad, selección del software, funcionalidad cliente-servidor, configuración del escanner, análisis y selección de la colección de planos a digitalizar, considerar el uso de formato JPG, que garantiza almacenamiento sin pérdida de información y permite trabajar la imagen en AutoCAD, así como el registro de datos y metadatos.

Es decir, los aspectos técnicos mencionados ameritan interrelacionarse de forma integral como estrategia para soportar, no sólo el control y recuperación de la información, sino también garantizar opciones de búsquedas, accesibilidad multiusuaria, capacidad de almacenamiento para altos volúmenes de información, visualización y descarga de los documentos técnicos.

\section{Técnicas de valoración y selección de documentos técnicos}

Como parte fundamental e integral de la gestión de documentos, se considera la selección y valoración de documentos, este proceso amerita concebirse para poder diferenciar las series que ameritan ser conservadas de aquellas que han de eliminarse. Por lo que logra aplicarse el Plan de Prescripción Documental PPDOC (2002) diseñado para tales efectos, que permite de acuerdo con las características intrínsecas y extrínsecas determinar los lapsos de temporalidad y vigencia de los documentos. Asimismo, se vale de las técnicas de muestreo para seleccionar aquellos documentos que de acuerdo a prescripciones previamente definidas dada la relevancia de los documentos, han de conservarse parcialmente de forma permanente. 


\section{Estandarización de sistemas y procesos de gestión}

La aplicación y efectividad de las técnicas archivísticas para la gestión integral de las colecciones documentales, ameritan ser asumidas como parte de los procesos estratégicos y preliminares para dar inicio a la sistematización de la colección de documentos técnicos (planos). Esta actividad, forma parte de la generación de valor agregado para estandarizar las formas de recuperación de la información.

Es por ello, que se hace referencia de las principales técnicas archivísticas cuya operatividad han permitido la estandarización del proceso de catalogación según los criterios que se exponen a continuación, al igual que permite el registro de inventario de la colección existente:

- Número de Registro: identificación en el estricto orden numérico secuencial, el cual es el que será utilizado para efectos del control de registro automatizado en el Sistema de Información a desarrollar. Facilita el control estricto que amerita establecerse para inventario y automatización simultánea del fondo técnico documental.

- Descripción del Plano: identificar si es un plano arquitectónico, urbanístico, estructural, paisajismo, de electricidad, aguas blancas, aguas negras, de redes. Asimismo, se refleja si la obra es una remodelación, ampliación, nuevos desarrollos, entre otros.

- Área de aplicación: identificar a que área de facultad, núcleo o dependencia pertenece.

- Fecha de elaboración: indicar el día, mes y año en el cual fue elaborado el plano.

- Tipo de Formato: amerita registrarse si el formato se encuentra en papel o en formato digital (se sugiere en el renglón de Observaciones indicar el tipo de papel).

- Tamaño: registrar las medidas del plano en función de centímetros.

- Condiciones del formato: previa evaluación del documento técnico (planos) seleccionar la opción que corresponda una vez evaluado y se determine si está en condiciones óptimas para su preservación, si posee o no condiciones de conservación o si en su defectos su condición de deterioro o contaminación amerita seleccionarse la opción de deterioro o su eliminación.

- Observaciones: para los efectos de previsión se sugiere colocar en este ítem si el documento amerita de forma inmediata pasar por procesos de Restauración o Eliminación.

Los datos considerados, cuyo formato se presenta en tabla 1, prevén accesibilidad tanto de forma manual como automatizada previa elaboración de los registros correspondiente.

Tabla 1 Modelo de formato para registro y catalogación de Planos

\begin{tabular}{|c|c|c|c|c|c|c|c|c|c|}
\hline $\begin{array}{c}\text { Registro } \\
\text { No. }\end{array}$ & $\begin{array}{l}\text { especialidad } \\
\text { del plano }\end{array}$ & $\begin{array}{c}\text { Lugar de } \\
\text { aplicación }\end{array}$ & $\begin{array}{l}\text { Fecha de } \\
\text { diseño }\end{array}$ & $\begin{array}{l}\text { Tipo de } \\
\text { formato }\end{array}$ & Tamaño & \multicolumn{3}{|c|}{ Condición } & Observación \\
\hline & & & & & & $\mathbf{P}$ & C & D & \\
\hline & & & & & & & & & \\
\hline
\end{tabular}

Fuente: Elaboración propia (2015)

Leyenda ( P: Preservado C: Conservado D: Deteriorado) 
Asimismo, garantiza la aplicación del procedimiento para llevar a efecto la catalogación, donde la efectividad del registro de datos permitirá de manera simultánea llevar a efecto el inventario de la colección existente, tanto de forma manual como automatizada, previa aplicación del Sistema de Clasificación Documental Administrativo (SICDA) concebido según Paredes y otros (1995), como el instrumento que garantiza, así como también ha logrado estandarizar la clasificación de documentos administrativos generados en la Universidad del Zulia, bajo estándares de normalización, lo cual permite dar solución a los problemas de organización, ordenación, clasificación y ordenación generada producto de las funciones básicas de las diferentes facultades y dependencias de LUZ.

El Sistema de Clasificación Documental Administrativo (SICDA) está estructurado por tres secciones, cada una de ellas se presentan en listas ordenadas por orden alfabético y por códigos:

- Sección Asuntos: Esta sección facilita la identificación y agrupación de los términos o asuntos más utilizados en el procesamiento documental, de igual forma, se describen los asuntos generales y específicos.

- Sección Estructura Organizativa de LUZ: Esta sección contempla todas las Facultades, Núcleos, Departamentos, Secciones, Centros e Institutos de Investigación, que conforman la estructura organizativa de LUZ. Para la estructuración de esta sección se utilizaron los registros de códigos presupuestarios asignados por la Dirección General de Planificación de la Universidad del Zulia (DGPLANILUZ), asimismo, se asignaron nuevos códigos respetando la estructura previa, para identificar aquellas dependencias que no fueron registradas previamente, por no poseer disponibilidad presupuestaria, aspecto que permitió el crecimiento lógico y estandarizado de códigos para el proceso documental.

- Sección Organismos Externos a LUZ, Nacionales e Internacionales: la conformación de todos los organismos nacionales e internacionales con los cuales la Universidad del Zulia interactúa y mantiene relaciones.

La efectiva aplicación del SICDA, permite obtener las siguientes ventajas: uniformidad de criterios con relación al procesamiento de los fondos documentales; considerar como orden prioritario los asuntos temáticos que se manejan, lo cual se convertirá en una de las opciones específicas para recuperar la información; unificación de códigos para un asunto determinado; diversidad de asuntos que faciliten y amplíen el análisis documental, considerando que todas las unidades del sistema de información universitario manejan una temática institucional o documentos similares; precisión en la asignación de códigos por asuntos generales y específicos.

De igual forma, permite el análisis de cada uno de los términos seleccionados con el fin de minimizar y eliminar los posibles problemas de homonimia: palabras que siendo iguales por su forma tienen distinta significación, minimiza problemas de sinonímia: circunstancia de ser sinônimos dos o, más vocábulos, evita el uso de términos obsoletos: términos poco usados, entre otros.

Sin embargo, la tipología y caracterización de la colección objeto de análisis conllevó a la adaptación de un índice auxiliar del Sistema de Clasificación Documental Administrativo, concebido como el Sistema De Clasificación Documental Técnica (Sidotec). Sistema creado, para dar respuesta en concordancia con las características intrínsecas y extrínsecas de la serie: Documentos Técnicos, diseñado como un instrumento auxiliar del Sistema de Clasificación Documental Administrativo (SICDA).

De acuerdo con lo antes expuesto, el Sidotec, se concibió como instrumento auxiliar de clasificación permite determinar los asuntos (temáticos) por áreas de especialidad que tratan los documentos técnicos según su especialización y el ente productor, tales como: topografía, arquitectura, estructura, instalaciones sanitarias, instalaciones eléctricas e instalaciones especiales, entre otras. Asimismo, la colección de material cibernético conformada por aproximadamente 120 cds., como respaldo digital de proyectos y obras ejecutadas desde el 2004 hasta la fecha, han sido clasificados y ordenados considerando el origen y procedencia de los mismos es decir, por dependencia o facultad que lo generó.

Por otro lado, el procesamiento de la colección de catálogos consta aproximadamente de 480 trípticos informativos sobre material a utilizar en el desarrollo de proyectos u obras. Este material, ha sido procesado mediante una variante del Sidotec, aplicando clasificaciones acordes a las áreas de especificación como: edificaciones, puertas, ventanas, mobiliario, electricidad, plomería, etc. Sin dejar de destacar, la aplicación de la 
base de datos conformada por más de 9.000 fotografías de planos y su ficha técnica dentro de la unidad de gestión técnica ha dado celeridad al proceso de búsqueda de documentos técnicos como planos.

\section{Gestión técnico documental: una proyección integral para la eficiencia}

Como proyección del sistema integral de gestión documental, se requiere prever la interacción efectiva de los procesos de producción-organización-digitalización-preservación-usabilidad-visibilidad de la colección documental técnica, así como fortalecer las configuraciones de la conexión de intranet consolidado en la DINFRA, lo cual propicia escenarios para garantizar la accesibilidad de la producción técnico- informativo documental generado en LUZ.

En consecuencia, se especifican los preceptos a ser considerados en el marco del desarrollo de la propuesta para la sistematización y gestión integral de la colección técnica documental:

- conformación de un equipo multidisciplinario constituido por profesionales: Archivólogos, Tecnólogos, Arquitectos, Ingenieros, Dibujantes, Diseñadores, entre otros, quienes de forma coordinada han de monitorear y garantizar el éxito del desarrollo del proyecto.

- realizar el análisis, diseño y desarrollo para la elaboración del Sistema Integral de Información Técnico Documental con visión de integración al repositorio institucional.

- estandarización y aplicación de la fase inicial de gestión archivística previamente presentada: catalogación, clasificación, definición de palabras clave o descriptoresmetadatos para la efectiva recuperación de información.

- consideración de la vigencia legal, administrativa e histórica según lo establecido en el Plan de Prescripción Documental aprobado a través del Acuerdo Universitario 403 por el consejo Universitario Consejo Universitario de fecha 22/5/2002.

- asignación y dotación de los recursos tecnológicos, requeridos para hacer efectivo la compatibilidad entre hardware (plataforma tecnológica) y software (sistemas de información), según lo prevé la Norma ISO 26300 relacionada con el Open Documento, la cual establece separación del contenido o imagen (planos) de las palabras clave o metadato.

El diseño propuesto, en líneas generales se concibe como una estrategia de proyección que ha de contribuir y garantizar la ejecución de auditorías y control en búsqueda y recuperación de información para afianzar la eficiencia y oportunidad de respuesta en los servicios de información. De igual forma, se destaca la importancia de integrar al Sistema de los Documentos existentes (Planos) tanto en formato electrónico con los que se encuentran por digitalizar.

\section{Conclusiones}

La débil concepción de los beneficios e impactos que generan la efectiva gestión de documentos técnicos, incide no sólo, al momento de recuperar la información, sino también en la preservación/conservación de los acervos documentales considerados en diversos casos con recursos patrimoniales y por ende con el dinamismo de la gestión que cualquier organización desea alcanzar.

En consecuencia, para afianzar parte de la gestión ya consolidada en la Dinfra-LUZ, se proyecta en el marco de los principios de gestión organizacional, hacer efectiva la creación de la Unidad de Gestión Técnico Documental de Infraestructura, lo cual prevé afianzar la efectiva modernización de los procesos que redundará en el cumplimiento de normas y estándares internacionales como las Normas ISO que inciden en la efectiva gestión archivística.

Sin dejar de destacar, la importancia de estandarizar los principios técnico-procedimentales expuesta para garantizar el procesamiento de la colección desde su producción (sistematización y digitalización), así como también la preservación, conservación y perdurabilidad de la memoria técnica documental de LUZ, lo cual redundará en la efectividad de servicios de información presenciales o en línea según requerimientos y demandas de los usuarios de una institución centenaria que afianza su visión en un crecimiento armónico y articulado de su planta física. 


\section{Referencias}

International Council on Archivies (2000) Sectionon Architectural Records" Guide to the Archival Care of Architectural Records 19 th- 20 th centuries". Francia ICA.

Mendo, C. (2004). Consideraciones sobre el método en archivística. Documenta \& Instrumenta

Paredes, A. y otros (2004) Transformación Organizacional y Funcional del Sistema e Archivo e Información de la Universidad del Zulia. SAILUZ. Universidad del Zulia- Maracaibo-Venezuela

Paredes, A; Párraga, M.; Medina, M.; Fuenmayor, L. y Vargas, L. (1995). Manual del sistema de Clasificación Documental Administrativa. SAILUZ. Universidad del Zulia. Maracaibo-Venezuela

Paredes, A.; Párraga, M.; Bohórquez, T.; Méndez, R. (2002). Plan de Prescripción Documental. SAILUZ. Universidad del Zulia. Maracaibo-Venezuela

Zambrano, F. (2005) Las nuevas tecnologías de la información y la comunicación en la concepción de una pedagogía alternativa para la educación superior. Conferencia invitada presentada en el Congreso Internacional de Pedagogía Alternativa. UPEL-IPB. Barquisimeto. 


\section{Dados dos autores}

\section{Ana Judith Paredes Chacin}

Profesora Asociada Facultad de Humanidades y Educación. Universidad del Zulia. Postdoctora en Gerencia de las Organizaciones. Doctora en Ciencias Gerenciales. Universidad Rafael Belloso Chacín. Magister en Gerencia de Empresas Mención: Servicios Administrativos. Licda. en Bibliotecología y Archivología. Universidad del Zulia. Editora de Enl@ce: Revista Venezolana de información, Tecnología y Conocimiento. Directora del Sistema de Servicios Bibliotecarios y de Información. Universidad del Zulia. (Serbiluz). Miembro acreditada al Programa de Promoción al Investigador ONCTI.

anajudithparedes@gmail.com

\section{Neyda Paredes Chacín}

Magister en Ciencias de la Comunicación. Mención: Tecnologías de Información y Comunicación. Universidad del Zulia. Lcda. Bibliotecología y Archivología. Universidad del Zulia. Archivóloga responsable de la Unidad Operativa de Gestión Documental de la Dirección de Infraestructura de LUZ.

neydaparedes77148@gmail.com

Recebido - Received: 2015-08-30

Aceitado - Accepted: 2015-11-16

\section{$(\mathrm{cc}) \mathrm{EY}$}

This work is licensed under a Creative Commons Attribution 4.0

United States License.

\section{UILIS D-Sorke}

This journal is published by the University Library System of the University of Pittsburgh as part of its D-Scribe Digital Publishing Program and is cosponsored by the University of Pittsburgh Press. 